\title{
A study of the original orbits of "hyperbolic" comets
}

\author{
M. Królikowska* \\ Space Research Centre of the Polish Academy of Sciences, Bartycka 18A, 00-716 Warsaw, Poland \\ Received 7 March 2001 / Accepted 27 June 2001

\begin{abstract}
The problem of hyperbolic incoming orbits for single-apparition comets is investigated. In this context the effect of non-gravitational acceleration on cometary dynamics was analyzed for the sample of 33 "hyperbolic" comets. The orbital elements of each cometary orbit were determined by the least squares procedure based on positional observations. These osculating orbital elements serve as a basis for the determination of the incoming orbits. The new method of estimating the uncertainties of $(1 / a)_{\text {ori }}$ based on a statistical approach to the orbit determinations is applied. For sixteen comets it was possible to determine the non-gravitational effects and original "non-gravitational" orbits. We show that for almost all the comets with detectable non-gravitational effects the incoming barycentric orbits changed from hyperbolic to elliptical when the non-gravitational effects were included. However, it was also ascertained that excess velocity at great distance are not cancelled by the nongravitational acceleration itself. We show that the non-gravitational effects are responsible for small modifications of the osculating elements (mainly eccentricity), which are sufficient, however, to obtain the elliptic original orbits for comets recognized in the literature as hyperbolic.
\end{abstract}

Key words. solar system - comets - long-period - non-gravitational effects

\section{Introduction}

It is generally accepted that long-period comets (e.g. comets with periods greater than the traditional lower limit of 200 years) originate from the Oort cloud, from which their stable orbits have been perturbed into the inner Solar System by the cumulative effects of the galactic tidal field and the passage of nearby stars or giant molecular clouds. Such comets possess nearly parabolic incoming orbits described by positive reciprocals of semimajor axes, e.g.: $(1 / a)_{\text {ori }}>0$.

However, in the sample of about three hundred long-period comets with well determined orbits there are about $10 \%$ which have hyperbolic incoming orbits $\left((1 / a)_{\text {ori }}<0\right)$. The problem of the negative tail of the distribution of original binding energies (proportional to $\left.(1 / a)_{\text {ori }}\right)$ has been discussed in detail in literature by many authors. First, the quality of astrometric data, the method of data selection (including the problem of weighting the individual measurements), and the applied method of integration may all strongly affect the orbit computation of each individual "hyperbolic" comet (even comets with orbits ascribed as 1A - highest quality class). Second, since all the negative excess energies are small, corresponding to roughly $-10^{-4} \mathrm{AU}^{-1}$ in inverse semimajor axis, Marsden et al. (1973) speculate that neglecting the non-gravitational effects tends to produce original orbit which appear to be more hyperbolic than they really are.

* e-mail: mkr@cbk.waw.pl
In particular, it is possible that most "hyperbolic" comets with small perihelion distances originate from the Oort's cloud of comets. The latter suggestion is strongly supported by the fact that perihelion distances of two comets with a greatest negative excess of $(1 / a)_{\text {ori }}$ are smaller than 0.9 AU. On the other hand, Yabushita (1991) found that the maximum excess velocity at great distance due to non-symmetric outgassing from cometary nuclei is equal to $0.18 \mathrm{~km} \mathrm{~s}^{-1}$. Thus, he argues that for at least 15 comets the calculated original orbits are "highly hyperbolic" and are not "compatible with elliptical orbits modified by the non-gravitational accelerations". More recently, Bolatto et al. (1995) concluded that the non-gravitational perturbation in a near-parabolic comet's energy per orbital revolution is generally smaller than about $10^{-4} \mathrm{AU}^{-1}$. However these two papers neglect the influence of variations of osculating orbital elements on $(1 / a)_{\text {ori }}$ when the non-gravitational effects are implied from observational data. Present calculations show that hyperbolic original orbits are produced by hyperbolic osculating orbits resulting from positional data in the case of pure gravitational motions.

There are several generally accepted reasons that extrasolar comets exist. An important question is whether such comets could be observed if they actually were observed. The modern theories (e.g. Clube \& Napier 1984; Duncan et al. 1987) for the formation of comets in the interstellar environment predict many more extrasolar comets than bound comets. However, the estimates of 
the expected rate for detection of extrasolar comets are uncertain. McGlynn \& Chapman (1989) show that the rate of hyperbolic comets passing with perihelion $q<2$ $\mathrm{AU}$ is 0.6 per year which implies that a total of about six extrasolar comets should have been detected over the last 150 years. More recently, Sen \& Rana (1993) argued that the density of stars in the solar neighbourhood was overestimated by McGlynn and Chapman. Using a value of $0.014 M_{\odot} \mathrm{pc}^{-3}$ Sen \& Rana found that the expected number of detectable interstellar comets per century is less than one. Hughes (1991) gives an even smaller number of expected comets on hyperbolic orbits. He argues that hyperbolic comets occur at a rate of $0.00225 \mathrm{yr}^{-1}$, which yields one such comet on average every $450 \mathrm{yrs}$. It seems clear that at most only a few known "hyperbolic" comets could really have interstellar origins. Nevertheless, using modern techniques for comet searches, the detection rate of extrasolar comets will increase dramatically in the future. Presently it is possible to weed out false members from the sample of "hyperbolic" comets.

In this paper the non-gravitational motions of individual "hyperbolic" comets from the Catalogue of Cometary Orbits (Marsden \& Williams 1997; hereafter MW Catalogue) are examined. The aim of the present study is to prove that incoming orbits of "hyperbolic" comets for which it was possible to determine the non-gravitational effects are almost all elliptical. Next, we show that using objective statistical criteria for the remaining "hyperbolic" comets, excess energies derived for pure gravitational motion are systematically smaller than those given in the MW Catalogue. The new method of $(1 / a)_{\text {ori }}$ uncertainty estimates are also used.

\section{The sample of "hyperbolic" comets}

In their catalogue Marsden \& Williams (1997) listed 307 comets with periods longer than 200 yrs and with well-determined orbits, e.g. comets belonging to quality classes 1 or 2 originally defined by Marsden et al. (1978). In this sample, 29 comets have negative reciprocals of original semimajor axes: $(1 / a)_{\text {ori }}<0$. Furthermore, 16 longperiod comets with determined non-gravitational effects and unknown $(1 / a)_{\text {ori }}$ (in the MW Catalogue) were taken into account. It turned out, however, that only six of these 16 comets had negative $(1 / a)_{\text {ori }}$. Thus, our sample consists of 35 candidates that have hyperbolic incoming orbits. Since the original observations were not available for four comets of the sample (1849 G2 Goujon; 1959 O1 Bester-Hoffmeister; 1895 W1 Perrine; 1911 S3 Beljawsky) the present analysis includes 31 comets observed before 1997 (see Tables 1 and 2). This sample was appended by two "hyperbolic" comets discovered after 1997.

\section{Original and future orbit determinations}

The orbital elements of each comet were recomputed on the basis of archive observations available at the Minor Planet Center (Cambridge, USA), with some additional data selected from the literature. For comets observed during the period of 1900-1950 the data collected in Warsaw in cooperation with Slovakian astronomers at the Astronomical Institute from Bratislava and Tatranska Lomnica were also taken into account. The observations used for the orbit determination in the present work are more complete than the data used in the MW Catalogue (see Col. 5 of Tables 1 and 2) except for two comets: 1898 V1 (Chase) and 1955 O1 (Honda). The observations for each comet were selected according to the objective criteria elaborated by Bielicki \& Sitarski (1991).

It has been shown that barycentric elements of the orbit determined at a distance above 150-200 AU change insignificantly (Todorovic-Juchniewicz 1981). For this reason each comet is followed from its position at a given epoch (see Table 3) backwards (original orbit) and forwards (future orbit) until the comet reaches a distance of $250 \mathrm{AU}$ from the Sun. The barycentric orbital parameters of incoming comets (before planetary perturbations) and outgoing comets (whose orbits have been perturbed) are called "original" and "future" quantities, respectively. In the numerical calculations the equations of motion are integrated in barycentric coordinates using recurrent power series method (Sitarski 1989) taking into account the perturbations by all nine planets. The respective values of original, osculating and future reciprocals of semimajor axis are given in Cols. 2-4 of Tables 1 and 2. The first line for each comet gives respective values of reciprocals of semimajor axis taken from the MW Catalogue.

The most hyperbolic original orbit in the sample was Comet Sato $1975 \mathrm{X} 1\left((1 / a)_{\text {ori }}=-1071\right.$ in units of $10^{-6} \mathrm{AU}^{-1}$ used in this paper) with $q=0.86$, and Comet Honda 1955 O1 $\left((1 / a)_{\text {ori }}=-635\right)$ with $q=0.89$ (see Table 1). In the next section we show that both these comets have strongly positive values of $(1 / a)_{\text {ori }}$ if the nongravitational solutions are considered.

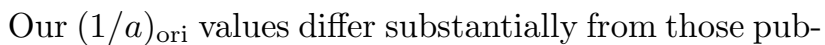
lished by Marsden and Williams in their catalogue. These discrepancies in $(1 / a)_{\text {ori }}$ are caused ${ }^{1}$ by an enlargement of the observational data and by adoption of the objective data selection procedure (Bielicki \& Sitarski 1991). The influence of data selection is seen for the first and third comet of Table 2 where the more restrictive selection procedure results in less negative values of $(1 / a)$ ori for these comets. Generally, the present $(1 / a)$ ori values are less negative than those given in the MW Catalogue (see Tables 1 and 2). Out of seventeen comets listed in Table 2, roughly half (nine comets) turned out to have positive $(1 / a)_{\text {ori }}$ values and none had a more negative value of $(1 / a)_{\text {ori }}$. Four comets with more negative values of $(1 / a)$ ori are given in Table 1 , but for all of them the non-gravitational effects change the original orbits from hyperbolical to elliptical (see the next section).

\footnotetext{
${ }^{1}$ If starting osculating values of orbital elements have been taken directly from the MW Catalogue the same values of $(1 / a)_{\text {ori }}$ were obtained (exact to one millionth of a $\mathrm{AU}^{-1}$ ) for all examined comets.
} 
Table 1. Reciprocals of semimajor axis for original, osculating and future orbits (in units of $10^{-6} \mathrm{AU}^{-1}$ ) for pure gravitational motion and for motion with non-gravitational effects represent by radial, $A_{1}$, and transverse, $A_{2}$, and - in two cases - normal, $A_{3}$, components. The "original" and "future" values of reciprocal of the semimajor axis are given in barycentric coordinates. The tenth column gives the mean original $(1 / a)$ ori obtained from a sample of 500 randomly selected orbits (with the mean rms residual given in the last column).

\begin{tabular}{|c|c|c|c|c|c|c|c|c|c|c|c|}
\hline \multirow{3}{*}{$\begin{array}{l}\text { Comet } \\
\text { design. } \\
\& \\
q \\
\\
1975 \text { V2 } \\
0.219\end{array}$} & & $\begin{array}{l}\text { Sta } \\
1 / a \\
\text { units o } \\
-6 \mathrm{AU}^{-} \\
\text {osc }\end{array}$ & ndard a & $\begin{array}{l}\text { non-gravitation } \\
\text { Interval of } \\
\text { observations }\end{array}$ & $\begin{array}{l}1 \text { orb } \\
\text { No. } \\
\text { of } \\
\text { obs. }\end{array}$ & $\begin{array}{l}\text { ts det } \\
\text { No. } \\
\text { of } \\
\text { res. }\end{array}$ & $\underset{\mathrm{rms}}{\mathrm{rmine}}$ & $\begin{array}{l}\text { from observ } \\
\text { Model }\end{array}$ & $\begin{array}{l}\text { tions } \\
\text { Non-gravitational } \\
\text { parameters } \\
A_{1} ; A_{2} ;\left(A_{3}\right) \\
\quad \text { (in units of } \\
\left.10^{-8} \mathrm{AU} \text { day }{ }^{-2}\right)\end{array}$ & $\begin{array}{l}\text { Sample of } \mathbf{5 0} \\
\left\langle(1 / a)_{\text {ori }}\right\rangle\end{array}$ & $\begin{array}{l}\text { dom orbits } \\
\text { Fitting } \\
\text { to obs. } \\
\text { with } \\
\text { rms of }\end{array}$ \\
\hline & -56 & -7 & 0 & $19751113-19760126$ & 70 & & & MW97 & & & \\
\hline & $\begin{array}{l}-148 \\
+453\end{array}$ & $\begin{array}{r}-99 \\
+503\end{array}$ & $\begin{array}{l}1125 \\
1728\end{array}$ & $\begin{array}{l}19751113-19760209 \\
19751113-19760209\end{array}$ & $\begin{array}{l}85 \\
85\end{array}$ & $\begin{array}{l}164 \\
164\end{array}$ & 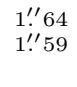 & $\begin{array}{l}\text { M1975V2 } \\
\text { M1975V2n }\end{array}$ & $\begin{array}{l}+1.599 \pm 4.372 \\
+0.5699 \pm .1642\end{array}$ & $\begin{array}{l}-148 \pm 23 \\
+408 \pm 822\end{array}$ & $\begin{array}{l}1 . \prime 71 \\
1 . \prime 65\end{array}$ \\
\hline $1956 \mathrm{R} 1$ & 0 & -531 & 0 & $19561108-19580411$ & 150 & & & MW97 & $+1.7 ;+0.1$ & & \\
\hline 0.316 & $\begin{array}{l}-104 \\
+118\end{array}$ & $\begin{array}{l}-785 \\
-562\end{array}$ & $\begin{array}{l}-636 \\
-387\end{array}$ & $\begin{array}{l}19561108-19580411 \\
19561108-19580411\end{array}$ & $\begin{array}{l}249 \\
249\end{array}$ & $\begin{array}{l}458 \\
458\end{array}$ & $\begin{array}{l}1 . \prime 78 \\
1 . \prime 55\end{array}$ & $\begin{array}{l}\text { M1956R1 } \\
\text { M1956R1n }\end{array}$ & $\begin{array}{l}+1.5169 \pm .1517 \\
+0.1170 \pm .0237\end{array}$ & $\begin{array}{r}-104.5 \pm 5.4 \\
+117.5 \pm 19.7\end{array}$ & $\begin{array}{l}1 . ! \\
1 ! \\
1.59\end{array}$ \\
\hline $1959 \mathrm{Y} 1$ & 0 & -288 & +0 & $19600104-19600617$ & 37 & & & MW97 & $+5.9 ;-1.4$ & & \\
\hline 0.504 & $\begin{array}{l}-135 \\
+186\end{array}$ & $\begin{array}{l}-589 \\
-268\end{array}$ & $\begin{array}{l}-586 \\
-265\end{array}$ & $\begin{array}{l}19600104-19600617 \\
19600104-19600617\end{array}$ & $\begin{array}{l}37 \\
37\end{array}$ & $\begin{array}{l}74 \\
74\end{array}$ & $\begin{array}{l}3 \stackrel{\prime \prime}{\prime \prime} 14 \\
1 . .47\end{array}$ & $\begin{array}{l}\text { M1959Y1 } \\
\text { M1959Y1n }\end{array}$ & $\begin{array}{r}+5.4803 \pm .4558 \\
-1.1497 \pm .3218\end{array}$ & $\begin{array}{l}-135 \pm 26 \\
+188 \pm 50\end{array}$ & $\begin{array}{l}3 . ! \\
1^{\prime \prime} 55\end{array}$ \\
\hline $\begin{array}{l}1989 \mathrm{Q} 1 \\
0.642\end{array}$ & $\begin{array}{r}0 \\
-.15\end{array}$ & $\begin{array}{r}-31 \\
-245\end{array}$ & $\begin{array}{r}+0 \\
+195\end{array}$ & $\begin{array}{l}19890824-19891224 \\
19890824-19891224\end{array}$ & $\begin{array}{l}180 \\
231\end{array}$ & 454 & $2 . \prime 03$ & $\begin{array}{c}\text { MW97 } \\
\text { M1989Q1 }\end{array}$ & $+3.4 ;+0.6$ & 1 & $1 \prime$ \\
\hline & +164 & -80 & +359 & $19890824-19891224$ & 231 & 454 & $1 .{ }^{\prime \prime} 78$ & M1989Q1n & $\begin{array}{l}+2.4160 \pm 0.7310 \\
+0.6677 \pm 0.2728\end{array}$ & $+161 \pm 52$ & $1 .^{\prime \prime} 83$ \\
\hline $1991 \mathrm{Y} 1$ & -94 & -90 & +1114 & 19911224-19920502 & 178 & & & MW97 & & & \\
\hline $\begin{array}{l}0.644 \\
1975 \times 1\end{array}$ & $\begin{array}{r}-98 \\
+6 \\
-734\end{array}$ & $\begin{array}{r}-94 \\
+10 \\
-1405\end{array}$ & $\begin{array}{l}+1111 \\
+1215 \\
-1461\end{array}$ & $\begin{array}{l}19911224-19920502 \\
19911224-19920502 \\
19751209-19760204\end{array}$ & $\begin{array}{c}274 \\
274 \\
59\end{array}$ & $\begin{array}{l}540 \\
540\end{array}$ & $\begin{array}{l}1 \stackrel{\prime \prime}{\prime \prime} 47 \\
1 . ! 41\end{array}$ & $\begin{array}{l}\text { M1991Y1 } \\
\text { M1991Y1n } \\
\text { MW97 }\end{array}$ & $+1.051 \pm 0.163$ & $\begin{array}{l}-98.1 \pm 9.3 \\
\quad+7 \pm 19\end{array}$ & $\begin{array}{l}1 .{ }^{\prime \prime} 50 \\
1 ., 44\end{array}$ \\
\hline & $\begin{array}{l}-1071 \\
+1037 \\
+967\end{array}$ & $\begin{array}{r}-1743 \\
+367 \\
+297\end{array}$ & $\begin{array}{r}-1701 \\
+309 \\
+239\end{array}$ & $\begin{array}{l}19751209-19760204 \\
19751209-19760204 \\
19751209-19760204\end{array}$ & $\begin{array}{l}82 \\
82 \\
82\end{array}$ & $\begin{array}{l}157 \\
157 \\
157\end{array}$ & $\begin{array}{l}22^{\prime \prime} 48 \\
22^{\prime \prime} 37 \\
22^{\prime \prime} 38\end{array}$ & $\begin{array}{c}\text { M1975X1 } \\
\text { M1975X1n1 } \\
{[\mathrm{M} 1975 X 1 \mathrm{n} 2]}\end{array}$ & $\begin{array}{l}+8.3477 \pm 2.1851 \\
+8.2386 \pm 2.2067 \\
+0.7452 \pm 1.745\end{array}$ & $\begin{array}{l}-1051 \pm 230 \\
+1048 \pm 599 \\
+896 \pm 647\end{array}$ & $\begin{array}{l}2^{\prime \prime}, 57 \\
2^{\prime \prime} 47 \\
2{ }^{\prime \prime} 47\end{array}$ \\
\hline $1955 \mathrm{O} 1$ & -727 & -1071 & -432 & $19550730-19551112$ & 102 & & & MW97 & & & \\
\hline 0.885 & $\begin{array}{r}-635 \\
+2002\end{array}$ & $\begin{array}{r}-978 \\
+1661\end{array}$ & $\begin{array}{r}-339 \\
2295\end{array}$ & $\begin{array}{l}19550802-19551113 \\
19550802-19551113\end{array}$ & $\begin{array}{l}60 \\
60\end{array}$ & $\begin{array}{l}105 \\
105\end{array}$ & $\begin{array}{l}1 \stackrel{\prime \prime}{15} \\
0 . \prime 91\end{array}$ & $\begin{array}{l}\text { M1955O1 } \\
\text { M1955O1n }\end{array}$ & $\begin{array}{l}+6.2749 \pm 0.8157 \\
+1.6158 \pm 0.4874\end{array}$ & $\begin{array}{l}-635 \pm 158 \\
+1986 \pm 381\end{array}$ & $\begin{array}{l}1 ., 21 \\
0.96\end{array}$ \\
\hline 1996 N1 & -161 & -621 & +526 & 19960704-19961012 & 283 & & & MW97 & & & \\
\hline 0.926 & $\begin{array}{r}-160 \\
-96\end{array}$ & $\begin{array}{l}-620 \\
-556\end{array}$ & $\begin{array}{l}+527 \\
+590\end{array}$ & $\begin{array}{l}19960704-19961103 \\
19960704-19961103\end{array}$ & $\begin{array}{l}316 \\
316\end{array}$ & $\begin{array}{l}614 \\
614\end{array}$ & $\begin{array}{l}0 . \prime \prime 81 \\
0, .81\end{array}$ & $\begin{array}{c}\text { M1996N1 } \\
\text { [M1996N1n] }\end{array}$ & $+0.9394 \pm 0.8936$ & $\begin{array}{c}-160.2 \pm 6.4 \\
-97 \pm 60\end{array}$ & $\begin{array}{l}0 . \prime 83 \\
0 !{ }^{\prime \prime} 83\end{array}$ \\
\hline $1998 \mathrm{P} 1$ & -128 & +205 & +1116 & 19980811-19990515 & 461 & 922 & $4 .^{\prime \prime} 68$ & M1998P1 & & $-128 \pm 10$ & $4 .^{\prime \prime} 75$ \\
\hline 1.15 & +797 & +1127 & +2036 & $19980811-19990515$ & 461 & 922 & $1 !^{\prime \prime} 18$ & M1998P1n & $\begin{array}{l}+32.611 \pm 0.417 \\
+0.8765 \pm .1368 \\
(-1.194 \pm .0549)\end{array}$ & $797 \pm 13$ & $1 !^{\prime \prime} 20$ \\
\hline 1968 N1 & -82 & -573 & +260 & $19680713-19681110$ & 131 & & & MW97 & & & \\
\hline 1.16 & $\begin{array}{r}-153 \\
+1728\end{array}$ & $\begin{array}{r}-644 \\
+1243\end{array}$ & $\begin{array}{r}+189 \\
+2072\end{array}$ & $\begin{array}{l}19680713-19681110 \\
19680713-19681110\end{array}$ & $\begin{array}{l}147 \\
147\end{array}$ & $\begin{array}{l}291 \\
291\end{array}$ & $\begin{array}{l}1: \prime 19 \\
1 . \prime 18\end{array}$ & $\begin{array}{l}\text { M1968N1 } \\
\text { M1968N1n }\end{array}$ & $\begin{array}{c}+5.697 \pm 3.001 \\
+15.406 \pm 7.061 \\
(-4.401 \pm 2.029)\end{array}$ & $\begin{array}{l}-147 \pm 117 \\
+1759 \pm 913\end{array}$ & $\begin{array}{l}1_{. \prime}^{\prime \prime} 22 \\
1_{.}^{\prime \prime} 22\end{array}$ \\
\hline 1986 P1 & 0 & -260 & & $19860805-19890411$ & 642 & & & MW97 & $+1.8 ;+0.1$ & & \\
\hline 1.20 & $\begin{array}{l}-.16 \\
+77 \\
+75\end{array}$ & $\begin{array}{l}-335 \\
-258 \\
-259\end{array}$ & $\begin{array}{l}+725 \\
+802 \\
+801\end{array}$ & $\begin{array}{l}19860805-19890411 \\
19860805-19890411 \\
19860805-19890411\end{array}$ & $\begin{array}{l}688 \\
688 \\
688\end{array}$ & $\begin{array}{l}1359 \\
1359 \\
1359\end{array}$ & 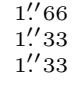 & $\begin{array}{c}\text { M1986P1 } \\
\text { M1986P1n1 } \\
{[\mathrm{M} 1986 \mathrm{P} 1 \mathrm{n} 2]}\end{array}$ & $\begin{array}{l}+1.7984 \pm 0.0655 \\
+1.7837 \pm 0.0672 \\
+.03329 \pm .03349\end{array}$ & $\begin{array}{l}-.14 \pm 1.02 \\
\quad+76 \pm 3 \\
\quad+76 \pm 3\end{array}$ & $\begin{array}{l}1 . \prime 69 \\
1 . ! 35 \\
1 . ' 35\end{array}$ \\
\hline $1971 \mathrm{E} 1$ & 0 & -628 & +0 & 19710309-19710909 & 131 & & & MW97n & $+5.6 ;-2$ & & \\
\hline 1.23 & $\begin{array}{r}-87 \\
+372 \\
+447\end{array}$ & $\begin{array}{l}-766 \\
-306 \\
-231\end{array}$ & $\begin{array}{l}-433 \\
+27 \\
+101\end{array}$ & $\begin{array}{l}19710309-19710909 \\
19710309-19710909 \\
19710309-19710909\end{array}$ & $\begin{array}{l}138 \\
138 \\
138\end{array}$ & $\begin{array}{l}275 \\
275 \\
275\end{array}$ & $\begin{array}{l}1^{\prime \prime}, 92 \\
1_{1}^{\prime \prime} 30 \\
1^{\prime \prime} 30\end{array}$ & $\begin{array}{c}\text { M1971E1 } \\
\text { M1971E1n1 } \\
{[\mathrm{M} 1971 \mathrm{E} 1 \mathrm{n} 2]}\end{array}$ & $\begin{array}{l}+6.2140 \pm 0.3548 \\
+6.3224 \pm 0.3744 \\
+0.5885 \pm 0.6348\end{array}$ & $\begin{array}{r}-87 \pm 76 \\
+375 \pm 58 \\
+447 \pm 101\end{array}$ & $\begin{array}{l}1^{\prime \prime} 97 \\
1.334 \\
1 ! .34\end{array}$ \\
\hline $\begin{array}{l}1996 \mathrm{E} 1 \\
1.35\end{array}$ & $\begin{array}{l}-42 \\
-41\end{array}$ & $\begin{array}{l}-654 \\
-652\end{array}$ & $\begin{array}{l}+356 \\
+357\end{array}$ & $\begin{array}{l}19960315-19961012 \\
19960315-19961012\end{array}$ & $\begin{array}{l}216 \\
249\end{array}$ & 494 & 1..06 & $\begin{array}{l}\text { MW97 } \\
\text { M1996E1 }\end{array}$ & & $-40.9 \pm 4.3$ & $1 . .09$ \\
\hline & $\begin{array}{r}-22 \\
+8\end{array}$ & $\begin{array}{l}+52 \\
+82\end{array}$ & $\begin{array}{l}+377 \\
+406\end{array}$ & $\begin{array}{l}19960315-19961012 \\
19960315-19961012\end{array}$ & $\begin{array}{l}249 \\
249\end{array}$ & $\begin{array}{l}494 \\
494\end{array}$ & 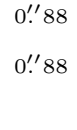 & $\begin{array}{l}\text { M1996E1n1 } \\
\text { [M1996E1n2] }\end{array}$ & $\begin{aligned}+6.333 & \pm 0.469 \\
+0.4137 & \pm 0.1544 \\
+7.169 & \pm 1.093 \\
-0.0358 & \pm 0.5521 \\
(+0.291 & \pm 0.343)\end{aligned}$ & $\begin{array}{c}-21.3 \pm 9.0 \\
+10 \pm 39\end{array}$ & $\begin{array}{l}0 . \prime 90 \\
0 !^{\prime \prime} 90\end{array}$ \\
\hline $\begin{array}{l}1946 \mathrm{C} 1 \\
1.72\end{array}$ & $\begin{array}{r}-13 \\
-4\end{array}$ & $\begin{array}{l}-678 \\
-669\end{array}$ & $\begin{array}{l}+373 \\
+382\end{array}$ & $\begin{array}{l}19460202-19470809 \\
19460129-19470809\end{array}$ & $\begin{array}{l}183 \\
498\end{array}$ & 807 & $2{ }^{\prime \prime} 92$ & $\begin{array}{l}\text { MW97 } \\
\text { M1946C1 }\end{array}$ & & & $2^{\prime \prime} 97$ \\
\hline & $\begin{array}{r}-4 \\
+36\end{array}$ & -628 & +423 & $19460129-19470809$ & 498 & 807 & & M1946C1n & $\begin{array}{l}+2.242 \pm 0.853 \\
+2.552 \pm 0.632\end{array}$ & $+36.6 \pm 8.1$ & 2 .' 89 \\
\hline 1993 A1 & $\begin{array}{r}0 \\
-18\end{array}$ & -918 & +0 & 19930102-19940610 & 539 & & & MW97n & $+16.6 ;-2.2$ & & \\
\hline 1.94 & $\begin{array}{r}-18 \\
+126\end{array}$ & $\begin{array}{r}-1066 \\
-922\end{array}$ & $\begin{array}{l}-539 \\
-395\end{array}$ & $\begin{array}{l}19921126-19940817 \\
19921126-19940817\end{array}$ & $\begin{array}{l}723 \\
723\end{array}$ & $\begin{array}{l}1446 \\
1446\end{array}$ & $\begin{array}{l}2^{\prime \prime \prime} 81 \\
1^{\prime \prime}, 20\end{array}$ & $\begin{array}{l}\text { M1993A1 } \\
\text { M1993A1n }\end{array}$ & $\begin{array}{c}+15.961 \pm 0.200 \\
-2.2460 \pm 0.2374\end{array}$ & $\begin{array}{l}-18.6 \pm 3.8 \\
+125.6 \pm 2.6\end{array}$ & 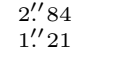 \\
\hline $\begin{array}{l}1946 \mathrm{U} 1 \\
2.41\end{array}$ & $\begin{array}{r}-1 \\
+3 \\
+65\end{array}$ & $\begin{array}{l}-393 \\
-389 \\
-327\end{array}$ & $\begin{array}{r}+26 \\
+107 \\
+92\end{array}$ & $\begin{array}{l}19461102-19481002 \\
19461101-19481002 \\
19461101-19481002\end{array}$ & $\begin{array}{c}97 \\
143 \\
143\end{array}$ & $\begin{array}{l}260 \\
260\end{array}$ & $\begin{array}{l}2 . \prime 09 \\
2 \text {.' }^{\prime \prime}\end{array}$ & $\begin{array}{l}\text { MW97 } \\
\text { M1946U1 } \\
\text { M1946U1n }\end{array}$ & $\begin{array}{l}+18.866 \pm 7.795 \\
+34.829 \pm 9.600 \\
\end{array}$ & $\begin{array}{l}+3.2 \pm 7.8 \\
+65 \pm 16\end{array}$ & $\begin{array}{l}2 . \prime 15 \\
2 . \prime 08\end{array}$ \\
\hline
\end{tabular}

The most positive value of $(1 / a)_{\text {ori }}=+4677$ belongs to Comet 1940 S1 Okabayashi-Honda (Table 2) whose orbit was calculated on the basis of 40 positional observations spanning a three months period. This orbit was poorly determined (the worst of whole sample), and significantly different from that given in the MW catalogue which was mainly caused by the three last observations of January 3rd, 1940. According to Kresak (1992) estimates 
Table 2. Reciprocals of semimajor axis for incoming, osculating and future orbits (in units of $10^{-6} \mathrm{AU}^{-1}$ ) for pure gravitational motion. The "original" and "future" values of reciprocal of the semimajor axis are given in barycentric coordinates. The eighth column gives the mean original $(1 / a)$ ori obtained from a sample of 500 randomly selected orbits (with the mean rms residual given in the last column).

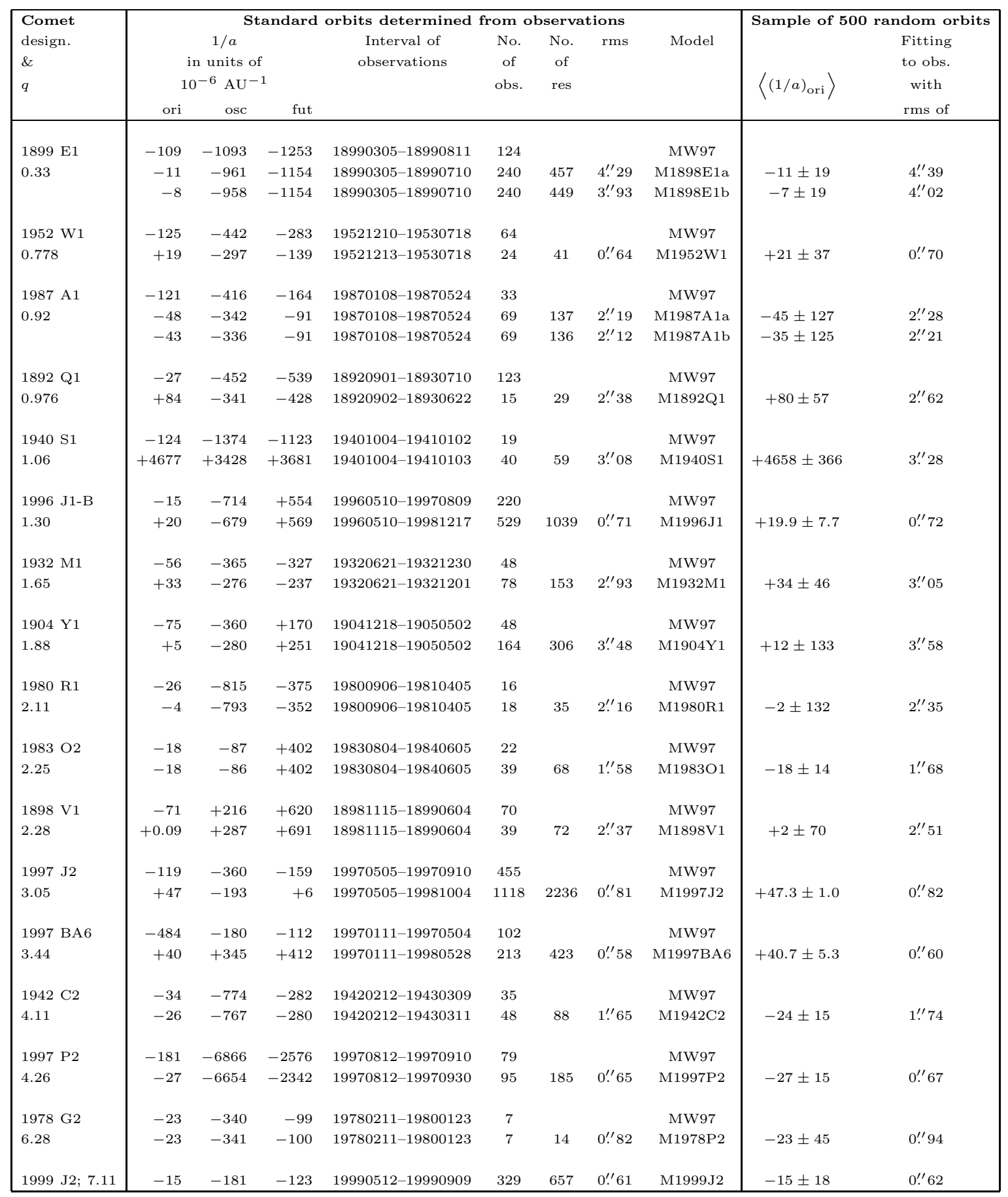

of internal errors in $1 / a$ for orbits of quality classes $1 \mathrm{~A}-$ $2 \mathrm{~B}$, this comet does not belong even to the lowest quality class $2 \mathrm{~B}$.

We have estimated the uncertainties of $(1 / a)_{\text {ori }}$ values. This was made by means of the following numerical simulations. The orbital elements (hereafter standard, or nominal elements) were determined by an iterative, least squares process from equations based on the positional observations. This "best solution" orbit is one of the potential orbits allowed by observations of the comet. Since we do not know the true cometary orbit, we will construct a set of randomly selected orbits which all fit well with the observations used for the orbit determination. The orbit selection procedure was adopted from Sitarski (1998) where the entire method was described in details. According to this procedure, we randomly selected a set 
of 500 orbits belonging to the same celestial body, e.g., a series of orbits in which the comet "could" move. A random number generator with Gaussian distribution was applied to select random values of the right sides of the elimination equations described in Sect. 3 of Sitarski's paper (1998). Then new values of orbital elements were calculated from the elimination equations. Each set of six orbital elements describing the chosen orbit differs from the nominal values, but they always fit the observations with an accuracy defined by residuals calculated according to Sitarski's method. The mean rms residuals of randomly selected orbits are given in the last column of Tables 1 and 2. Next, for each random orbit we calculated the original orbit. Thus, for each comet we obtained the distribution of original reciprocals of semimajor axes (Figs. 1 and 2). The normal distribution was then fitted to the histogram of original $1 / a_{\text {ori }}$.

Examples of the Gaussian function fitting to the obtained $(1 / a)_{\text {ori }}$ distributions are given in Fig. 1 for Comet 1997 P2 (Spacewatch), and in Fig. 2 for three other comets. In this way we calculated the mean value of $1 / a_{\text {ori }}$ and its standard deviation $\sigma$. The goodness of fit was tested by a $\chi^{2}$ method and fitting by normal distributions was very good in all cases. The respective values of $\left\langle 1 / a_{\text {ori }}\right\rangle$ with their $\sigma$ 's are given in Tables 1 and 2 . One finds that the planetary perturbations also contribute to the calculated uncertainties. However, for all the comets from the sample the uncertainties in original and future orbits are similar to uncertainties in randomly selected orbits and reflect the internal errors of orbital elements derived from astrometric data. This is illustrated in Fig. 1 for Comet 1997 P2 (Spacewatch) which underwent the strongest energy perturbation (among the group of analyzed objects) during its passage through the planetary system. Before its discovery this comet passed close to Jupiter (at a distance of about $0.65 \mathrm{AU}$ ) and in the middle of 2000 it passed 2.7 AU from Saturn losing $\delta(1 / a)=(1 / a)_{\text {fut }}-(1 / a)_{\text {ori }}=-2315$ (see Table 2$)$, mostly due to these two close encounters. Thus, this comet suffers planetary perturbations of almost a factor of 4 greater than the typical (mean) value of energy perturbations of long-period comets (about $\delta(1 / a)=670$; Yabushita 1979). For all 500 randomly selected orbits we obtained, the calculated uncertainties of $(1 / a)_{\text {fut }}$ and $\delta(1 / a)$ are equal to 36 and 21 , respectively. This means that the observational uncertainties usually do not affect our estimates of planetary perturbations and do not contribute substantially to the uncertainties of calculated $(1 / a)_{\text {ori }}$ and $(1 / a)_{\text {fut }}$. Therefore, uncertainties of $(1 / a)_{\text {ori }}$ given in Tables 1 and 2 are closely connected with the orbital quality classes $1 \mathrm{~A}-$ $2 \mathrm{~B}$.

\section{Non-gravitational effects}

Traditionally, the term "non-gravitational effect" has been reserved for the outgassing acceleration of the comet nucleus caused by asymmetric sublimation of cometary volatiles. The method to determine non-gravitational effects on the orbital motion of a comet was proposed by Marsden et al. (1973). In the commonly accepted formalism the three orbital components of the non-gravitational acceleration acting on a comet are symmetric relative to perihelion and can be written as:

$$
\begin{aligned}
& F_{i}=A_{i} g(r), \quad A_{i}=\text { const for } i=1,2,3 \\
& g(r)=\alpha\left(r / r_{0}\right)^{-2.15}\left[1+\left(r / r_{0}\right)^{5.093}\right]^{-4.6142} \\
& r_{0}=2.808, \alpha=0.111262
\end{aligned}
$$

where $F_{1}, F_{2}, F_{3}$ represent the radial, transverse and normal components of the non-gravitational acceleration, respectively. The analytical function $g(r)$ simulates the ice sublimation rate as a function of the heliocentric distance $r$ and was based on an empirical water sublimation curve given by Delsemme \& Miller (1971). The nongravitational effects play an important role in the vast majority of short-period comets. At least three consecutive apparitions are needed to precisely determine the independent non-gravitational parameters $A_{1}, A_{2}, A_{3}$ within an investigated time interval. This means that for oneapparition comets these effects will be poorly determined. However, a greater difficulty was pointed out by Yabushita (1991, 1996). He showed that the non-gravitational accelerations of some nearly parabolic comets could be inconsistent with the above physical model of the comet nucleus if only $\mathrm{H}_{2} \mathrm{O}$ sublimation was assumed (represented by the function $g(r)$ ). In particular, he argued that for the Comet 1989 Q1 the recoil due to outgassing of $\mathrm{H}_{2} \mathrm{O}$ is too small (almost by a factor of 10) to be compatible with the observed value of the non-gravitational acceleration unless the nuclear density is as low as $0.1 \mathrm{~g} \mathrm{~cm}^{-3}$ and nucleus diameter is smaller than $4 \mathrm{~km}$. There are strong reasons to suppose that the sublimation from nearly parabolic comets may be controlled by $\mathrm{CO}$ or $\mathrm{N}_{2}$ molecules rather than the less volatile $\mathrm{H}_{2} \mathrm{O}$ molecules. It seems especially true for comets with perihelia distances outside of $3 \mathrm{AU}$, where carbon monoxide production significantly exceeds production of $\mathrm{H}_{2} \mathrm{O}$. Moreover, coming "fresh" from the Oort cloud these comets should exhibit a much higher content of the most volatile ices (e.g. $\mathrm{CO}, \mathrm{CO}_{2}$ ) that are substantially or completely depleted during its first perihelion passage.

Thus, Yabushita proposed a convenient form for the dependence of acceleration on the heliocentric distance based on the assumption that the sublimating molecules are $\mathrm{CO}$ instead $\mathrm{H}_{2} \mathrm{O}$ :

$F_{i}=A_{i}^{*} f(r), \quad A_{i}^{*}=\mathrm{const} \quad$ for $\quad i=1,2,3$,

$f(r)=2.75 / r^{2} \cdot 10^{-0.22185(r-1) / 3} \cdot\left(1+0.0006 r^{5}\right)^{-1}$.

These two formulae for non-gravitational accelerations (Eqs. (1) and (2)) are considered in the present investigation. 


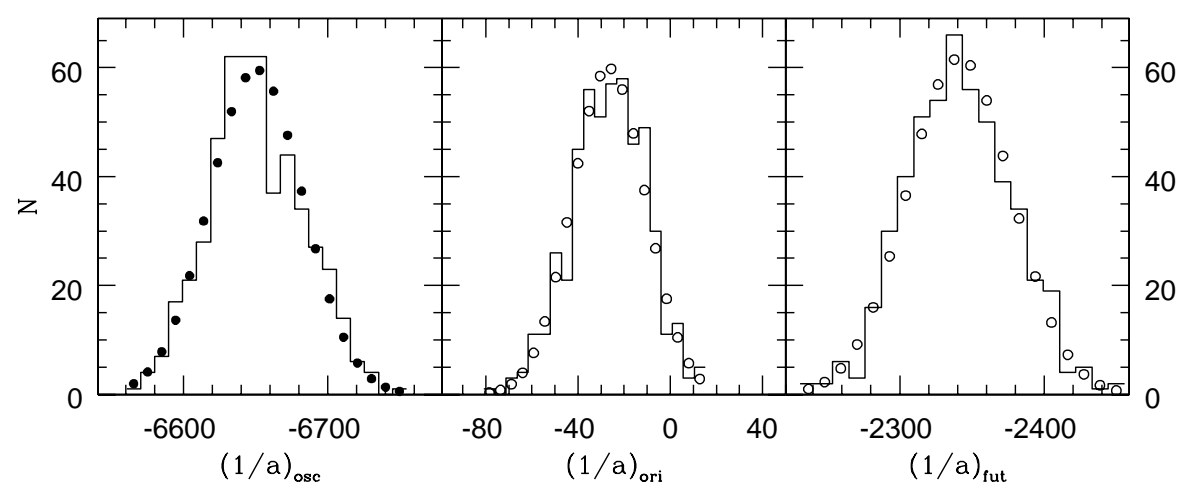

Fig. 1. Distributions of reciprocals of semimajor axes for Comet 1997 P2 (Spacewatch). The left panel shows distribution of heliocentric $(1 / a)_{\text {osc }}$ for 500 dynamical clones of the osculating orbit (from the normal distribution given by black dots) which were then integrated backwards and forwards to the distance of 250 AU from the Sun. In this way the distributions of $(1 / a)_{\text {ori }}$ and $(1 / a)_{\text {fut }}$ for barycentric orbits were derived and these are shown in the middle and in the right panels, respectively.

The Gaussian functions fitted to the $(1 / a)_{\text {ori }}$ and $(1 / a)_{\text {fut }}$ distributions are represented by open circles.
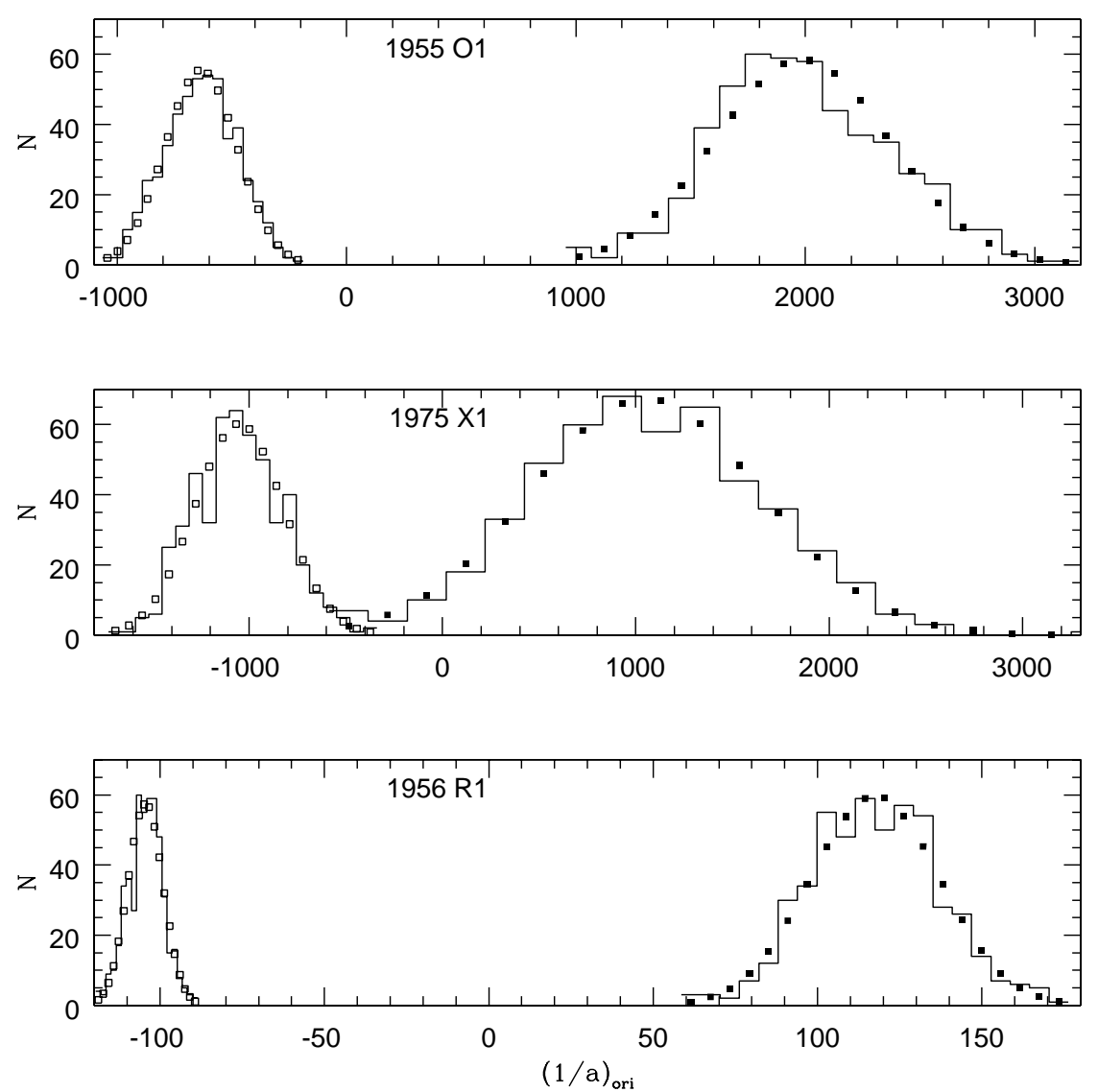

Fig. 2. Distribution of original reciprocals of semimajor axes $(1 / a)_{\text {ori }}$ (in units of $10^{-6} \mathrm{AU}^{-1}$ ) derived from integrations of 500 dynamical clones of the osculating orbit of three comets. In each panel, the left histogram corresponds to the pure gravitational solution and the right one - to the non-gravitational solution. The Gaussian functions fitted to the $(1 / a)_{\text {ori }}$ distributions are shown with squares; the mean values of Gaussian distributions $\left\langle(1 / a)_{\text {ori }}\right\rangle$ and their uncertainties are given in the tenth column of Table 1.

\subsection{Standard non-gravitational effects due to $\mathrm{H}_{2} \mathrm{O}$ sublimation}

For sixteen comets from the sample it was possible to obtain the non-gravitational solutions due to outgassing of $\mathrm{H}_{2} \mathrm{O}$ volatile molecules from the cometary surface. For five of them only the radial component of the non-gravitational force was determined; for the remaining comets the radial, $A_{1}$, and transverse, $A_{2}$, components were determined with reasonable accuracies (and 
in two cases also the normal, $A_{3}$, component). Starting from such "non-gravitational" solutions the original "nongravitational" orbits could be calculated.

Results of the new determinations of the $(1 / a)_{\text {ori }}$, taking into account the non-gravitational effects, are presented for 16 comets in Table 1 . The models given in the square brackets represent non-gravitational parameters determined with poor accuracy. Distributions of original reciprocals of semimajor axes for two comets (1975 X1 Sato and 1955 O1 Honda) with the most hyperbolic original orbits in the MW Catalogue, and for a comet with small perihelion distance $q \simeq 0.32$ (1956 R1 ArendRoland) are shown in Fig. 2.

One can see (Table 1) that in almost all cases, the original orbits changed from hyperbolic to elliptical when the non-gravitational effects represented by $A_{1}, A_{2}$ (and $\left.A_{3}\right)$ parameters were included. This means that the $(1 / a)_{\text {ori }}$ become positive for the "non-gravitational" orbits. The most interesting solution was obtained for Comet 1975 X1 Sato, the most "hyperbolic" comet from the MW Catalogue. For this comet only $A_{1}$ could be determined with a reasonably small error ${ }^{2}$. However, it seems that $A_{1}$ plays an important role in the orbit determination for this comet, changing the osculating orbit from open to closed, which - as a consequence - generates an elliptic original orbit, too. The analogous tendency is evident for the next three solutions with $A_{1}$ alone (M1986P1n1, M1971E1n1, and M1991Y1n); only in the case of Comet $1996 \mathrm{~N} 1$ is the $(1 / a)_{\text {osc }}$ modification insufficient to change the original orbit from open to close curve.

Only two negative values of "non-gravitational" $(1 / a)_{\text {ori }}$ are present in Table 1 . The first is quoted above, Comet Brewington $1996 \mathrm{~N} 1$, for which it was possible to obtain only $A_{1}$ and is presented only to show that the non-gravitational solution does not help in this case. In the considered group of comets potentially exposed to the action of the non-gravitational effects this one seems to be the best candidate for an interstellar object. The second exception was Comet 1996 E1 NEAT. For this best quality osculating orbit (1A class in the MW Catalogue) we derived a slightly hyperbolic original orbit in the pure gravitational solution. Next, we considered two "nongravitational" solutions: with two $\left(A_{1}\right.$ and $\left.A_{2}\right)$ and with all three non-gravitational parameters (Model M1996E1n1 and Model M1996E1n2, respectively). In the first case the original orbit still seems to be hyperbolic, but with a slightly smaller absolute value of $(1 / a)_{\text {ori }}$. For the second non-gravitational solution the original orbit became elliptical, but its $(1 / a)_{\text {ori }}$ value is very small. This second solution indicates that if non-gravitational effects really play an important role in the motion of Comet 1996E1 (which is suggested by the substantial decrease of the residuals), the observational data are insufficient to

\footnotetext{
${ }^{2}$ For comparison we present the second non-gravitational solution where also $A_{2}$ was taken into account, however the uncertainty of $A_{2}$ determination is larger than the value $A_{2}$ itself.
}

determine all three non-gravitational parameters, e.g. the full "non-gravitational" solution. The analogous calculations for the remaining comets from Table 1 were performed, and in all cases where the value of $A_{3}$ parameter was determinable, osculating and original orbits were more elliptical than those given in Table 1, but like in the Comet $1996 \mathrm{E} 1$ case, $A_{3}$ determinations were very poor. Only the full "non-gravitational" solution for two comets (1998 P1 Williams, 1968 N1 Honda) seem to be realistic, and for this reason are given in Table 1.

The present calculations confirm the Marsden et al. (1978) hypothesis that original orbits of comets of small and moderate $q$ appear to be negative due to disregard of the non-gravitational forces during calculations. It is important to stress that influence of the non-gravitational effects on $(1 / a)_{\text {ori }}$ is caused by different orbital elements (in particular eccentricity) derived for the non-gravitational solutions and gravitational solutions on the basis of observational data. The case of Comet 1975 X1 Sato is an adequate example (Table 3 ). The non-gravitational perturbations in the comet's energy per orbital revolution are significantly smaller than $100 \times 10^{-6} \mathrm{AU}^{-1}$ for all the considered comets, which is in agreement with previous results (e.g. Yabushita 1991; Weissman 1979; Bolatto et al. 1995).

\subsection{Non-gravitational effects caused by $\mathrm{CO}$ sublimation}

Finally, we examined how the function $f(r)$ (Eq. (2)) approximates the non-gravitational accelerations of all the sixteen comets with detectable non-gravitational effects. Generally, it turned out that the "non-gravitational" orbits for comets sublimating $\mathrm{CO}$ volatile molecules from their surfaces can be fit to observational data with the same residuals as orbits for comets undergoing the $\mathrm{H}_{2} \mathrm{O}$ sublimation, however the $A_{2}^{*} / A_{1}^{*}$ ratios substantially changed in some cases. Comet 1946 U1 is a good example: in the model $1946 \mathrm{U} 1 \mathrm{n}$ the $A_{2}$ parameter dominated over $A_{1}$ almost by a factor of two, whereas in the model 1946U1c both components $\left(A_{1}^{*}\right.$ and $\left.A_{2}^{*}\right)$ are comparable. The orbital elements and values of $(1 / a)_{\text {ori }}$ are also fairly similar. Therefore, in Table 4 the non-gravitational determinations of $(1 / a)_{\text {ori }}$ are presented for some representative cases only. One can see that for only two comets (models 1998P1 c and 1993A1 c), the non-gravitational parameters based on $\mathrm{CO}$ sublimation are a better fit to observations than those based on standard $\mathrm{H}_{2} \mathrm{O}$ sublimation.

\section{Summary}

The main conclusions can be summarized as follows:

1. Dividing the present sample of the long-period comets which hypothetically are of interstellar origin in two subgroups according to criterion of $q<2.5 \mathrm{AU}$ and $q>2.5 \mathrm{AU}$, we have 27 members $(82 \%)$ of the former group and only $6(18 \%)$ of the latter. This reflects 
Table 3. Original reciprocals of semimajor axes, perihelion distances and eccentricities for Comet 1975 X1 Sato. Osculating orbits were determined from observational data in the pure gravitational case (Models A and B), and in the non-gravitational case (Models C and D). These two kinds of osculating orbits were integrated backwards without the non-gravitational parameters (Models $\mathrm{A}$ and $\mathrm{C}$ ) and with the non-gravitational parameter $A_{1}$ consistent with non-gravitational osculating solution (Models B and D). Thus, Model A is the same as M1975X1, and Model D is the same as M1975X1n1 (see Table 1).

\begin{tabular}{|c|c|c|c|c|c|c|}
\hline Model & Year & $(1 / a)_{\text {ori }}\left[\mathrm{AU}^{-1}\right]$ & $q_{\text {ori }}[\mathrm{AU}]$ & $e_{\text {ori }}$ & $A_{1}\left[\mathrm{AU}\right.$ day $\left.^{-2}\right]$ & $(1 / a)_{\mathrm{osc}}\left[\mathrm{AU}^{-1}\right]$ \\
\hline A & 1689 & -.00107090 & 0.86122673 & 1.00092229 & 0.0 & -.001743 \\
\hline B & 1689 & -.00107101 & 0.86122708 & 1.00092239 & $8.2386 \times 10^{-8}$ & -.001743 \\
\hline $\mathrm{C}$ & 1665 & +.00103740 & 0.86119984 & 0.99910659 & 0.0 & +.000367 \\
\hline $\mathrm{D}$ & 1665 & +.00103744 & 0.86119960 & 0.99910655 & $8.2386 \times 10^{-8}$ & +.000367 \\
\hline
\end{tabular}

Table 4. The same as in Table 1 but with the non-gravitational effects due to CO sublimation.

\begin{tabular}{|c|c|c|c|c|c|c|c|c|}
\hline \multicolumn{7}{|c|}{ Non-gravitational orbits determined from observations } & \multirow{2}{*}{\multicolumn{2}{|c|}{$\begin{array}{r}\text { Sample of } 500 \text { random orbits } \\
\text { Fitting } \\
\text { to obs. }\end{array}$}} \\
\hline \multirow[t]{3}{*}{ Model } & \multirow{2}{*}{\multicolumn{3}{|c|}{$\begin{array}{c}1 / a \\
{[\text { in units of }} \\
\left.10^{-6} \mathrm{AU}^{-1}\right]\end{array}$}} & \multirow[t]{3}{*}{ rms } & \multicolumn{2}{|c|}{$\begin{array}{c}\text { Non-gravitational } \\
\text { parameters }\end{array}$} & & \\
\hline & & & & & $A_{1}^{*}$ & $A_{2}^{*}\left(A_{3}^{*}\right)$ & $\left\langle(1 / a)_{\text {ori }}\right\rangle$ & with \\
\hline & ori & osc & fut & & [in units of 10 & ${ }^{-8} \mathrm{AU}_{\mathrm{day}^{-2}}{ }^{-2}$ & & rms of \\
\hline M1956R1 c & +120 & -560 & -385 & $1^{\prime \prime} .55$ & $+1.184 \pm .115$ & $+0.0744 \pm .0189$ & $+121 \pm 18$ & $1^{\prime \prime} .59$ \\
\hline M1959Y1 c & +192 & -262 & -260 & $1^{\prime \prime} 47$ & $+3.983 \pm .328$ & $-0.796 \pm .236$ & $+194 \pm 51$ & $1^{\prime \prime} 55$ \\
\hline M1989Q1 c & +165 & -80 & +359 & $1^{\prime \prime} .79$ & $+1.798 \pm 0.542$ & $+0.500 \pm 0.198$ & $+166 \pm 51$ & 1.. 83 \\
\hline M1975X1 c & +1035 & +365 & +307 & $2 . .37$ & $+6.137 \pm 1.607$ & & $+1068 \pm 603$ & $2^{\prime \prime} .47$ \\
\hline M1955O1 c & +2083 & +1742 & 2377 & 0.'91 & $+4.677 \pm 0.609$ & $+1.150 \pm 0.348$ & $+2083 \pm 391$ & $0^{\prime \prime} 96$ \\
\hline M1998P1 c & +642 & +973 & 1883 & 1.' 10 & $+18.615 \pm 0.211$ & $\begin{array}{c}+0.323 \pm 0.088 \\
(-0.460 \pm 0.031)\end{array}$ & $+642 \pm 10$ & $1^{\prime \prime} 11$ \\
\hline M1971E1 c & +396 & -283 & +50 & 1.. 30 & $+4.148 \pm 0.254$ & $+0.245 \pm 0.440$ & $+399 \pm 102$ & $1^{\prime \prime} 34$ \\
\hline M1996E1 c & -97 & -23 & +301 & $0.0^{\prime \prime} 85$ & $+3.939 \pm 0.278$ & $+0.350 \pm 0.098$ & $-97 \pm 9$ & $0 . .87$ \\
\hline M1946C1 c & +35 & -629 & +422 & $2 . .85$ & $+1.277 \pm 0.314$ & $+1.087 \pm 0.320$ & $+35 \pm 8$ & $2^{\prime \prime} 90$ \\
\hline M1993A1 c & +137 & -911 & -383 & $1^{\prime \prime} 17$ & $+5.307 \pm 0.068$ & $-1.220 \pm 0.077$ & $+137 \pm 3$ & 1.'19 \\
\hline M1946U1 c & +64 & -328 & +91 & $2 . .02$ & $+2.992 \pm 0.765$ & $+2.355 \pm 0.955$ & $+65 \pm 16$ & $2 . .09$ \\
\hline
\end{tabular}

the trend visible in the full sample of long-period comets with well determined orbits where about $80 \%$ of comets have $q<2.5$.

2. The values of original semimajor axis (and values of orbital elements) are susceptible to selection criteria. For this reason the objective criteria described by Bielicki \& Sitarski (1991) are recommended. Using such criteria we found that 10 comets from the entire sample of 33 hyperbolic comets $(30 \%)$ have positive $(1 / a)_{\text {ori }}$ values (see Tables 1 and 2). We also showed that for the remaining "hyperbolic" comets, excess energies derived for pure gravitational motion are systematically smaller than those given in the MW Catalogue, with the exception of four cases. All four comets with more negative values of $(1 / a)_{\text {ori }}$ change their original orbits from hyperbolic to elliptic (see point 3) when non-gravitational effects are taken into account.

3. For 16 comets from the sample, non-gravitational solutions were obtained. In most cases the incoming barycentric orbits changed from hyperbolic to elliptic when the non-gravitational effects were included. There are only two exceptions to this rule. For both of them the tendency of $(1 / a)_{\text {ori }}$ to decrease is evident, but not enough to modify the shape of their orbit from an open to closed curve (see Table 1). This result is in formal disagreement with Yabushita (1991) who drew a conclusion that for all comets which have an original orbit with excess hyperbolic velocity (calculated under the assumption of pure gravitational motion) larger than $0.18 \mathrm{~km} \mathrm{~s}^{-1}$ it is impossible to explain its hyperbolicity in terms of non-gravitational effects. However, he neglected the fact that osculating orbital elements are changed when the non-gravitational effects are applied to observational data. Including non-gravitational effects into the orbit determination, we obtain osculating elements somewhat different from those determined with an assumption of gravitational motion alone. In particular, the pure gravitational osculating orbits are more hyperbolic than in the nongravitational cases, which have a direct influence on the shape of their original orbits. The present analysis reveals that these small modifications of the osculating elements (mainly eccentricity), are sufficient to obtain elliptic original orbits for comets which previously - under the assumption of pure gravitational motion - appeared to be 
"hyperbolic". It was also shown that all non-gravitational parameters, even $A_{1}$ alone, play an important role in orbit determination from positional observations.

4. Determining the cometary orbit from observational data in the pure gravitational case and in the nongravitational case, and then integrating both orbits backwards in time we obtained differences between original reciprocal semimajor axes $\Delta(1 / a)_{\text {ori }}$ of up to 2637 (in units of $10^{-6} \mathrm{AU}^{-1}$, see Table 1$)$. This means that nongravitational orbits could significantly affect the Oort peak, i.e. concentration of long-period comets in the range of $0<(1 / a)_{\text {ori }}<100 \times 10^{-6} \mathrm{AU}^{-1}$ which was previously missed in literature (e.g. Weissman 1985; Bolatto et al. 1995). All the authors considered the non-gravitational perturbation in comet's energy per orbital revolution (in fact significantly smaller than $100 \times 10^{-6} \mathrm{AU}^{-1}$ ), whereas the problem lies in different non-gravitational osculating orbits resulting from the observational data.

5 . It is difficult to judge which relation between the heliocentric distance and the acceleration (Eqs. (1) and (2)) better characterizes the non-gravitational trends in the astrometric data of one-apparition comets. To do this, additional photometric and spectroscopic data at great distances from the sun are needed. The "non-gravitational" orbits for comets sublimating CO volatile molecules from their surfaces are fit to astrometric data with almost the same residuals as orbits for comets undergoing $\mathrm{H}_{2} \mathrm{O}$ sublimation; only in two cases did the non-gravitational parameters based on $\mathrm{CO}$ sublimation give a slightly better fit to observations than those based on standard $\mathrm{H}_{2} \mathrm{O}$ sublimation.

6. Present analysis reduced the sample of 33 putative "hyperbolic" comets to only 10 objects with negative original reciprocals.
Acknowledgements. The author is deeply indebted to Professor Grzegorz Sitarski for helpful discussions on various aspects of this investigations. This work was supported by the Polish Committee of Scientific Research (the KBN grant 2.P03D.002.18).

\section{References}

Bielicki, M., \& Sitarski, G. 1991, Acta Astron., 41, 309

Bolatto, A. D., Fernandez, J. A., \& Carballo, G. F. 1995, Planet. Space Sci., 43, 709

Clube, S. V. M., \& Napier, W. M. 1984, MNRAS, 208, 575

Delsemme, A. H., \& Miller, D. C. 1971, Planet. Space Sci., 19, 1229

Duncan, M., Quinn, T., \& Tremaine, S. 1987, AJ, 94, 1330

Hughes, D. W. 1991, J. Br. Astron. Assoc., 101, 119

Królikowska, M., \& Sitarski, G. 1996, A\&A, 310, 992

Królikowska, M., Sitarski, G., \& Szutowicz, S. 1998, A\&A, 335, 757

Marsden, B. G., \& Sekanina, Z. 1973, AJ, 78, 1118

Marsden, B. G., Sekanina, Z., \& Yeomans, D. K. 1973, AJ, 78, 211

Marsden, B. G., Sekanina, Z., \& Everhart, E. 1978, AJ, 83, 64

Marsden, B. G., \& Williams, G. V. 1997, Catalogue of Cometary Orbits, twelfth edition

McGlynn, T. A., \& Chapman, R. D. 1989, AJ, 346, L105

Sekanina, Z. 1984, AJ, 89, 1573

Sekanina, Z. 1988, AJ, 96, 1455

Sen, A. K., \& Rana, N. C. 1993, A\&A, 275, 298

Sitarski, G. 1989, Acta Astron., 39, 345

Sitarski, G. 1998, Acta Astron., 48, 547

Todorovic-Juchniewicz, B. 1981, Acta Astron., 31, 191

Weissman, P. R. 1979, AJ, 84, 580

Weissman, P. R. 1985, Space Sci. Rev., 41, 299

Yabushita, S. 1979, MNRAS, 187, 445

Yabushita, S. 1991, Earth, Moon and Planets, 52, 87

Yabushita, S. 1996, MNRAS, 283, 347 